\title{
Advances in Materials
}

2017; 6(1-1): 10-17

$\mathrm{http}: / / \mathrm{www} . \mathrm{sciencepublishinggroup.com} / \mathrm{j} / \mathrm{am}$

doi: $10.11648 /$ j.am.s.2017060101.12

ISSN: 2327-2503 (Print); ISSN: 2327-252X (Online)

\section{Contact Around a Sharp Corner with Small Scale Plasticity}

\author{
Zupan $\mathrm{Hu}^{1,2}$ \\ ${ }^{1}$ Department of Mechanical Engineering, University of Michigan, Ann Arbor, USA \\ ${ }^{2}$ Stanley Black \& Decker Inc, Towson, USA
}

Email address:

zupanhu@umich.edu

To cite this article:

Zupan Hu. Contact Around a Sharp Corner with Small Scale Plasticity. Advances in Materials. Special Issue: Advances in Multiscale Modeling Approach. Vol. 6, No. 1-1, 2017, pp. 10-17. doi: 10.11648/j.am.s.2017060101.12

Received: October 31, 2016; Accepted: November 8, 2016; Published: December 8, 2016

\begin{abstract}
Owing to elastic singularity, the contact stress around a sharp corner is highly sensitive to the boundary conditions and local geometrical details. Determination of such stress is critical in predicting failures such as wear, fretting fatigue and crack initiation. In this paper, the stress around such corner is analyzed based on linear elasticity and small scale plasticity. The stress on the contact interface is generalized in a way that the results can be easily converted to represent another corner with different dimensions or boundary conditions. An example is presented to show the determination of the stress scale and the formulation of a generalized solution. It is shown that the generalized macro stress field away from the corner dominates the contact behaviors around the corner.
\end{abstract}

Keywords: Partial Slip, Stress Singularity, Plastic Yielding

\section{Introduction}

The stress field around a contact interface with a sharp corner is closely related to various failures such as wear, fatigue and plastic yielding, which significantly limit the life of various engineering components [1-14]. The analysis of contact stress around these area is important in understanding the onset and propagation of those failures: it will provide guidelines for the design of contact components so that the damage can be limited [11, 12, 15-21]. Based on experimental observations, the sliding behavior between two elastic bodies in contact can be described by three regimes on a map of tangential and normal loads: full-stick, partial-slip and gross-sliding regimes [22-24]. The damage is determined by the energy dissipation process in the slip regions $[22,25]$. Such identification is crucial for predicting the life of many engineering components. For instance, studies have shown that crack growth is accelerated greatly at the boundary between the slip and stick zones, reducing the fatigue life significantly [25-32].

If the two contacting components are assumed to be elastic bodies with a constant coefficient of friction, the state of stick or slip at the corner of the contact interface, where partial slip regimes are often located [22, 33-40], is determined by the coefficient of friction and the stress-intensity factors for the singular stresses at the corner [41]. The stress state at the corner of a solid attached to a half-plane is equivalent to that of a notch with the same corner angle [42, 43]. An in-depth analysis has shown that the contact stress between an elastic block and a half-plane can be treated as an eigenvalue problem [44]. The stress singularity at the corner has an exponent that is the same value for both the shear and normal compressive contract stresses, and is a function of the contact angle. For instance, a $90^{\circ}$ corner has an exponent of -0.4555 [45]. The ratio between the interfacial shear and normal stresses pressure is independent of the applied forces, and only depends on the geometry. This leads to a critical friction coefficient of 0.543 (for the $90^{\circ}$ corner) above which the interface sticks. Below this value of friction coefficient, slip will always occur at the corner even in the absence of an applied shear force [46].

In addition to the geometrical details, plasticity can affect frictional behavior in other ways. For example, yield can occur away from the interface. For example, below a critical normal load, yield occurs at the interface of a sphere on a flat surface, but it occurs below the contact region at higher loads [41], [47]. The effect of yielding can potentially significantly affect the stress field at the contact surface by relaxing the contact stresses. One methodology to study the plasticity of metals in microscale is to develop a statistical volume 
element (SVE) which takes into account the variability in the microstructure. Different from representative volume element (RVE) in which average value is assumed for all realizations, the SVE model can have a different value from one realization to another. SVE models have been developed to study polycrystalline microstructures. Zhang et al. developed a novel SVE model to improve computational efficiency compared to traditional RVE models [48]. The key grain features, including orientation, misorientation, grain size, grain shape, grain aspect ratio are considered in their SVE model. A slip-based damage criterion is developed to study the plasticity of aluminum alloy at the microscale [49]. Their study shows that the multiscale damage criterion can successfully characterize the microscale plasticity of FCC structure alloys and predict plasticity based damage evolution.

The goal of this paper is to investigate the effects of slip and small scale plasticity on the stress field on contacting surfaces, with a particular emphasis on the behavior at corners. By incorporating those contributions into the frictional analysis, it is expected that the stick and slip zones can be predicted more reliably, which will be important in subsequent investigation to model provide the much needed fundamentals for related studies such as fretting fatigue and wear. The investigations are done using a model configuration of a block on a flat surface, and comparing the slip behavior along the interface when Coulomb's law is assumed to the slip behavior when the effects of plasticity is included.

\section{Methodology}

\subsection{Parameters of the Model}

This study will focus on a commonly used contact setup: the plane stress model of a rectangular block in contact with a large substrate of the same material. As shown in Fig. 1, the bottom of the substrate is fixed in both the horizontal and vertical directions. It was confirmed that the substrate is large enough for boundary effects to be neglected for small scale plasticity (Slip zone or plastic zone size is less than $10 \%$ of the contact interface). The indenter is subjected to a uniformly distributed normal pressure load, $p$ and a uniformly distributed tangential load, $q$. The vertical load is applied first, as a preload, and then the tangential load is applied to investigate the slip behavior. The response at the contact interface depends on factors such as the magnitude of $p$ and $q$, the young's modulus $E$, and the dimensions of the system. The nomenclature used in this paper is given in Table 1 , and values of the non-dimensional parameters used in the simulations are given in Table 2.

Table 1. Nomenclature of the system.

\begin{tabular}{ll}
\hline$\tau y$ & Shear yield strength of the bulk material \\
$\tau \mathrm{i}$ & Contact shear stress (left is positive) \\
$\sigma \mathrm{i}$ & Contact compressive normal stress \\
$\mu$ & Friction coefficient \\
$\mathrm{E}$ & Young's modulus \\
$v$ & Poisson's ratio \\
$\mathrm{p}$ & Normal pressure load \\
$\mathrm{q}$ & Shear traction load \\
$\mathrm{x}$ & Distance from the left corner along the contact interface \\
$\mathrm{r}$ & Distance from the left corner along $\theta$ \\
$\beta$ & External wedge angle as shown in Fig. 1 \\
$\alpha$ & Symmetry angle as shown in Fig. $1 . \alpha=\pi-\beta / 2$ \\
$\theta$ & Angle from the symmetry line as shown in Fig. 1 \\
\hline
\end{tabular}

Table 2. Mechanical properties and boundary conditions in the simulations.

\begin{tabular}{ll}
\hline$v$ & 0.25 \\
$\mathrm{~h}_{1}$ & $10 \mathrm{~mm}$ \\
$\mathrm{p}$ & $10000 \mathrm{kPa}$ \\
$\mathrm{q}$ & $2500 \mathrm{kPa}$ \\
$\mathrm{E}$ & $7.5 \times 10^{7} \mathrm{kPa}$ \\
\hline
\end{tabular}

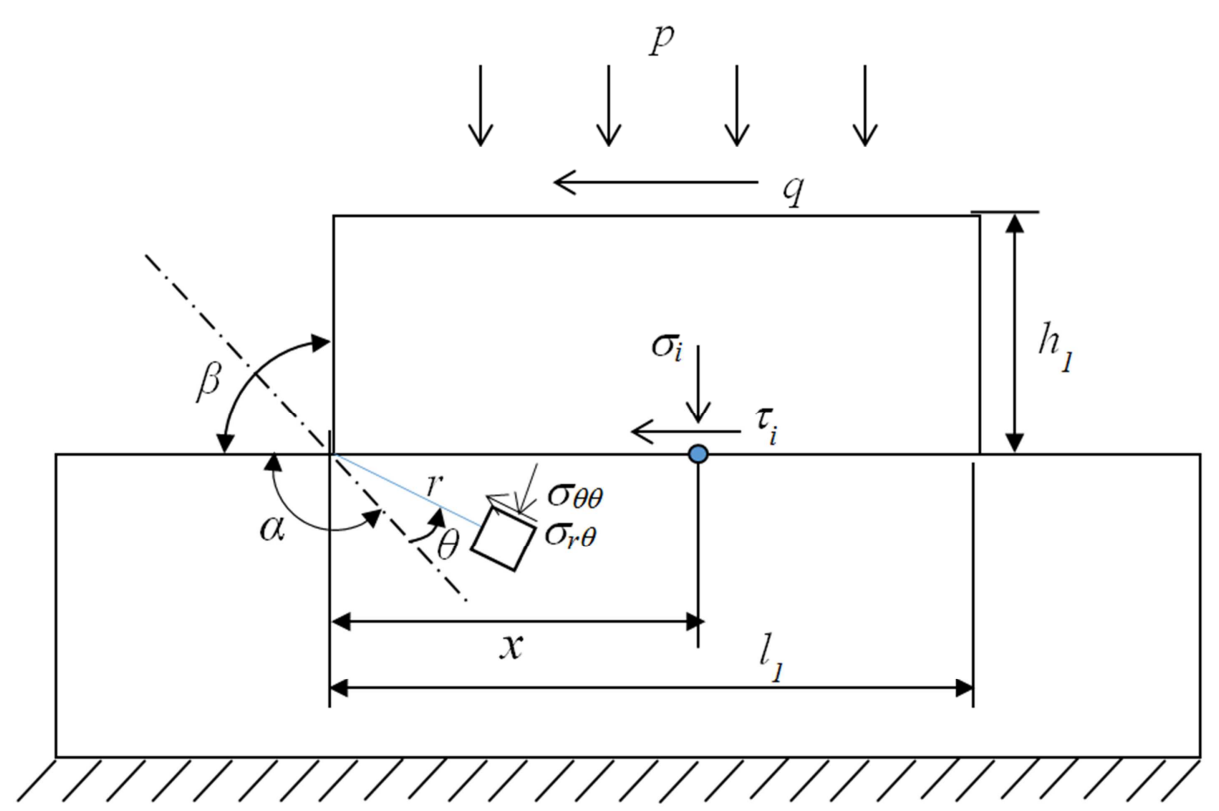

Figure 1. A representative contact model. The contact compressive normal stress $\sigma_{i}$ and shear stress $\tau$ are applied on the substrate surface, with positive directions defined as shown in the figure. 


\subsection{Friction Law}

Coulomb's friction law describes the relationship between the frictional force and the normal load by means of a constant friction coefficient. Based on this law, slip will occur when the condition as follows is satisfied,

$$
\left|\tau_{i}\right|=\mu \sigma_{i}
$$

where $\mu$ is the coefficient of friction, $\tau_{i}$ is the shear stress on the interface, $\sigma_{i}$ is the normal stress on the interface.

\subsection{Plastic Deformation}

We consider plasticity by assuming the material to be elastic / perfectly-plastic during the loading step. A von Mises yielding criteria is used, i.e. yielding will occur if the von Mises stress as shown below reaches the yielding strength.

$$
\sigma_{v}=\sqrt{\sigma_{x}^{2}-\sigma_{x} \sigma_{y}+\sigma_{y}^{2}+3 \sigma_{x y}^{2}}
$$

Here, the $\sigma_{x}$ and $\sigma_{y}$ are the stress components in $\mathrm{x}$ and $\mathrm{y}$ direction.

\section{Results}

\subsection{Scaling of the System}

A contact with little slip is elastically similar to the notch problem [50]. We can normalize the length and stress field of the system with the scales defined based on the solution of an infinite large wedge $[51,52]$ to make the results general. In such a case the detailed loading and geometry of the model are not important; what matters are the stress-intensity factors. We will also use the asymptotic stress solution as benchmark to validate our numerical simulations. When the slip is large, such a normalization approach does not offer a benefit anymore. Instead, we will use the physical dimension and applied shear load to normalize the length and stress.

The asymptotic stress solution of the notch problem can be written as [53]

$$
\begin{gathered}
\sigma_{\theta \theta}(r, \theta)=K_{I} f_{1}(\theta) r^{\lambda_{I}-1}+K_{I I} f_{2}(\theta) r^{\lambda_{I I}-1}, \\
\sigma_{r \theta}(r, \theta)=K_{I} f_{1}(\theta) g_{1}(\theta) r^{\lambda_{I}-1}+K_{I I} f_{2}(\theta) g_{2}(\theta) r^{\lambda_{I I}-1}
\end{gathered}
$$

where $K_{I}$ and $K_{I I}$ are the stress-intensity factors corresponding to the eigenvalues $\lambda_{I}$ and $\lambda_{I I}$ respectively, $r$ is the distance from the wedge corner, and $\theta$ is the angle shown in Fig. 1. The angular dependence is given by

$$
\begin{gathered}
f_{1}(\theta)=\frac{\cos \left[\left(\lambda_{I}+1\right) \theta\right] \cos \left[\left(\lambda_{I}-1\right) \alpha\right]-\cos \left[\left(\lambda_{I}-1\right) \theta\right] \cos \left[\left(\lambda_{I}+1\right) \alpha\right]}{\cos \left[\left(\lambda_{I}-1\right) \alpha\right]-\cos \left[\left(\lambda_{I}+1\right) \alpha\right]} \\
f_{2}(\theta)=\frac{\sin \left[\left(\lambda_{I I}+1\right) \theta\right] \sin \left[\left(\lambda_{I I}-1\right) \alpha\right]-\sin \left[\left(\lambda_{I I}-1\right) \theta\right] \sin \left[\left(\lambda_{I I}+1\right) \alpha\right]}{-\sin \left[\left(\lambda_{I I}-1\right) \alpha\right]+\frac{\lambda_{I I}-1}{\lambda_{I I}+1} \sin \left[\left(\lambda_{I I}+1\right) \alpha\right]},
\end{gathered}
$$

The functions $g_{1}(\theta)$ and $g_{2}(\theta)$ are given by

$$
\begin{array}{r}
g_{1}(\theta)=\frac{\sin \left[\left(\lambda_{I}+1\right) \theta\right]-\Gamma_{I}^{s} \sin \left[\left(\lambda_{I}-1\right) \theta\right]}{\cos \left[\left(\lambda_{I}+1\right) \theta\right]-\Gamma_{I}^{c} \cos \left[\left(\lambda_{I}-1\right) \theta\right]}, \\
g_{2}(\theta)=\frac{-\cos \left[\left(\lambda_{I I}+1\right) \theta\right]+\Gamma_{I I}^{c} \cos \left[\left(\lambda_{I I}-1\right) \theta\right]}{\sin \left[\left(\lambda_{I I}+1\right) \theta\right]-\Gamma_{I I}^{s} \sin \left[\left(\lambda_{I I}-1\right) \theta\right]}, \\
\text { where } \Gamma_{i}^{s}=\frac{\sin \left[\left(\lambda_{i}+1\right) \alpha\right]}{\sin \left[\left(\lambda_{i}-1\right) \alpha\right]} \text { and } \Gamma_{i}^{c}=\frac{\cos \left[\left(\lambda_{i}+1\right) \alpha\right]}{\cos \left[\left(\lambda_{i}-1\right) \alpha\right]}
\end{array}
$$

We define a length scale $x_{0}$ and a stress scale $\tau_{0}$ by

$$
\begin{gathered}
x_{0}=\left(\frac{K_{I}}{K_{I I}}\right)^{\frac{1}{\lambda_{I I}-\lambda_{I}}}, \\
\tau_{0}=\left(\frac{K_{I}^{1-\lambda_{I I}}}{K_{I I}^{1-\lambda_{I}}}\right)^{\frac{1}{\lambda_{I}-\lambda_{I I}}},
\end{gathered}
$$

Normalizing the stress fields by $x_{0}$ and $\tau_{0}$, Equations 3 and 4 become

$$
\begin{gathered}
\frac{\sigma_{\theta \theta}(r, \theta)}{\tau_{0}}=f_{1}(\theta)\left(\frac{r}{x_{0}}\right)^{\lambda_{I}-1}+f_{2}(\theta)\left(\frac{r}{x_{0}}\right)^{\lambda_{I I}-1}, \\
\frac{\sigma_{r \theta}(r, \theta)}{\tau_{0}}=f_{1}(\theta) g_{1}(\theta)\left(\frac{r}{x_{0}}\right)^{\lambda_{I}-1}+f_{2}(\theta) g_{2}(\theta)\left(\frac{r}{x_{0}}\right)^{\lambda_{I I}-1},
\end{gathered}
$$

Note that the normalized stresses do not depend on the dimensions of the system or on the loading conditions, which makes the analysis more general. Along the interface, these equations reduce to

$$
\begin{gathered}
\frac{\sigma_{c}(x)}{\tau_{0}}=f_{1}\left(\frac{\beta}{2}\right)\left(\frac{x}{x_{0}}\right)^{\lambda_{I}-1}+f_{2}\left(\frac{\beta}{2}\right)\left(\frac{x}{x_{0}}\right)^{\lambda_{I I}-1}, \\
\frac{\tau_{c}(x)}{\tau_{0}}=f_{1}\left(\frac{\beta}{2}\right) g_{1}\left(\frac{\beta}{2}\right)\left(\frac{x}{x_{0}}\right)^{\lambda_{I}-1}+f_{2}\left(\frac{\beta}{2}\right) g_{2}\left(\frac{\beta}{2}\right)\left(\frac{x}{x_{0}}\right)^{\lambda_{I I}-1},
\end{gathered}
$$

Note that Eqs. 13 and 14 are only valid for pure elastic analysis without any slip. For $\beta=\pi / 2, \lambda_{I}$ and $\lambda_{I I}$ are calculated to be 0.5445 and 0.9085 , respectively. The functions $g_{1}$ and $g_{2}$ are 0.543 and -0.219 , respectively on the interface. Therefore, the values of $K_{I}$ and $K_{I I}$, which depend on the loads and geometry, can be obtained by analyzing the stress field around the corner. We have built finite-element models (FEM) using ABAQUS. Figure 2 shows an example with 25881 elements. At the corner of the contact at the interface, where a singularity occurs, the element size is refined to be about $10^{11}$ smaller than that of 
the regular elements in order to capture the stress gradient. The possibility of slip is switched off for the purpose of calculating $K_{I}$ and $K_{I I}$, whose values are obtained by minimizing the $\chi$ function defined as

$$
\chi^{2}=\sum\left\{\sigma_{\theta \theta}(r, \theta)-\left[K_{I} f_{1}(\theta) r^{\lambda_{I}-1}+K_{I I} f_{2}(\theta) r^{\lambda_{I I}-1}\right]\right\}^{2},
$$

for all elements on a circle close to and around the corner, as shown in the bottom of Fig. 2.
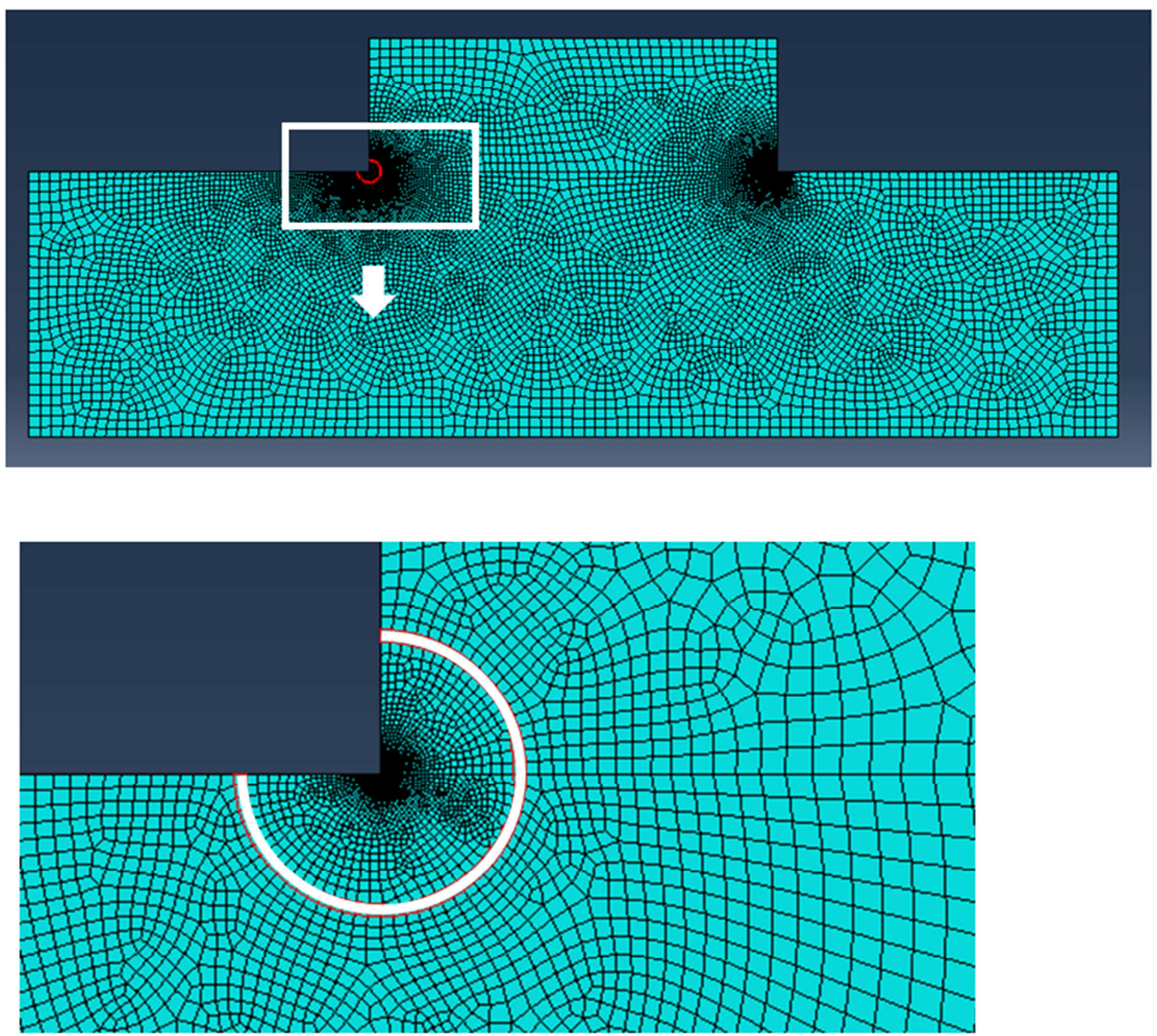

Figure 2. The finite element model for the simulations. The bottom figure shows a magnified view of the mesh around the corner.

\subsection{An Example to Determine Stress and Length Scales}

The loading conditions and dimensions in Figure 3 (a) and Table 2 are used to illustrate the procedure of determining the stress and length scales. When $x$ is small, the second term of Eq. (2) is negligible, and this equation can be rewritten as,

$$
\sigma_{\theta \theta}\left(r, \frac{\pi}{4}\right) \cong K_{I} f_{1}\left(\frac{\pi}{4}\right) x^{\lambda_{I}-1}=K_{I} f_{1}\left(\frac{\pi}{4}\right) r^{-0.4555},
$$

$K_{I}$ can be calculated by linear curve fitting of stress $\sigma_{\theta \theta}$ versus $r^{-0.4555}$. The stress components of a series of elements along the line with $\theta=\pi / 4$, as well as the distance to the left corner $r$, are computed and the value of $K_{I}$ can be obtained by the linear curve fitting shown in Figure 4.

Based on Figure 4, a relationship of $K_{I} f_{1}\left(\frac{\pi}{4}\right)=$
$7532.03 \pm 21.47 \mathrm{kPa}^{*} \mathrm{~mm}^{0.4555}$ is obtained. $f_{1}\left(\frac{\pi}{4}\right)$ can be calculated by Eq.(5), so the first stress intensity factor $K_{I}$ is $10314.3 \pm 29.1 \mathrm{kPa}^{*} \mathrm{~mm}^{0.4555}$

The second order intensity factor $K_{I I}$ becomes significant when the radius is relatively large, so it can be calculated by fitting the stress field of a circle region as is shown in the magnified view in Figure 2. Now Eq. (2) is rewritten as,

$$
\sigma_{\theta \theta}(r, \theta)-K_{I} f_{1}(\theta) r^{\lambda_{1}-1}=K_{I I} f_{2}(\theta) r^{\lambda_{I I}-1}
$$

$K_{I}, f_{2}(\theta)$ and $f_{1}(\theta)$ are known. So again $K_{I I}$ can be obtained through linear curve fitting of $\sigma_{\theta \theta}(r, \theta)-K_{I} f_{1}(\theta) r^{\lambda_{I}-1}$ versus $f_{2}(\theta) r^{\lambda_{I I}-1}$. As shown in Figure 5, $K_{I I}$ is found to be $4263.9 \pm 67.5 \mathrm{kPa}^{*} \mathrm{~mm}^{0.0915}$.

Under small slip conditions, the elastic solution dominates the mechanics outside the slip zone, so we then can use $x_{0}$ 
and $\tau_{0}$ to normalize the dimension and the contact stresses. Based on the $K_{I}$ and $K_{I I}$ obtained above and Eqs.(9) and (10), the length and stress scales are $x_{0} / l_{1}=1.44$ and $\tau_{0} / E=$
$4.55 \times 10^{-5}$. Using $x_{0}$ and $\tau_{0}$, a general form of stress field can obtained which can represent any loading conditions or dimensions that has the same corner angle. This generality will be illustrated in the next section.
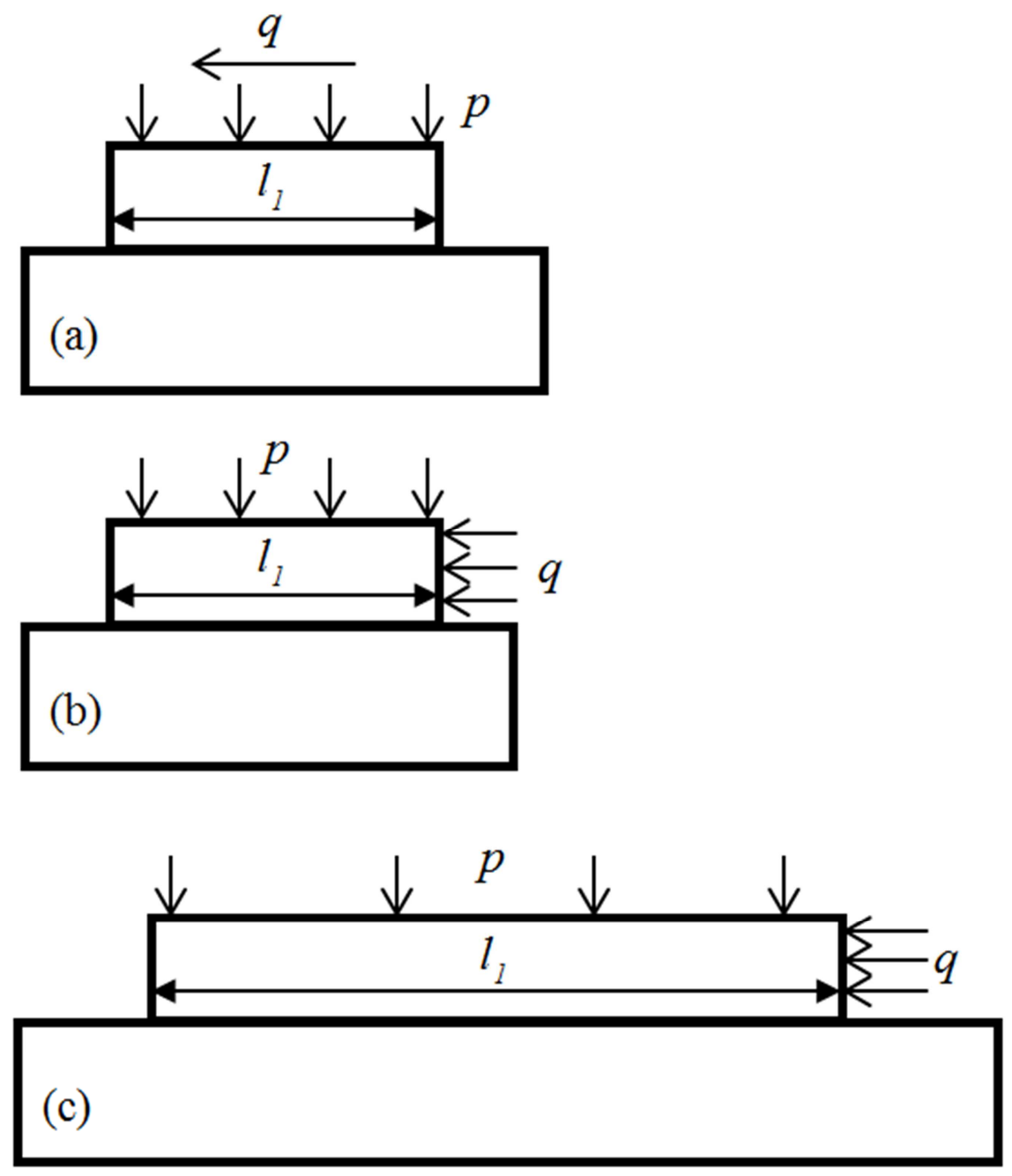

Figure 3. Various loading conditions and system dimensions. (a) Top loading with $l_{1} / h_{1}=3$ (b) Side loading with $l_{l} / h_{1}=3$ (c) Side loading with $l_{l} / h_{1}=9$.

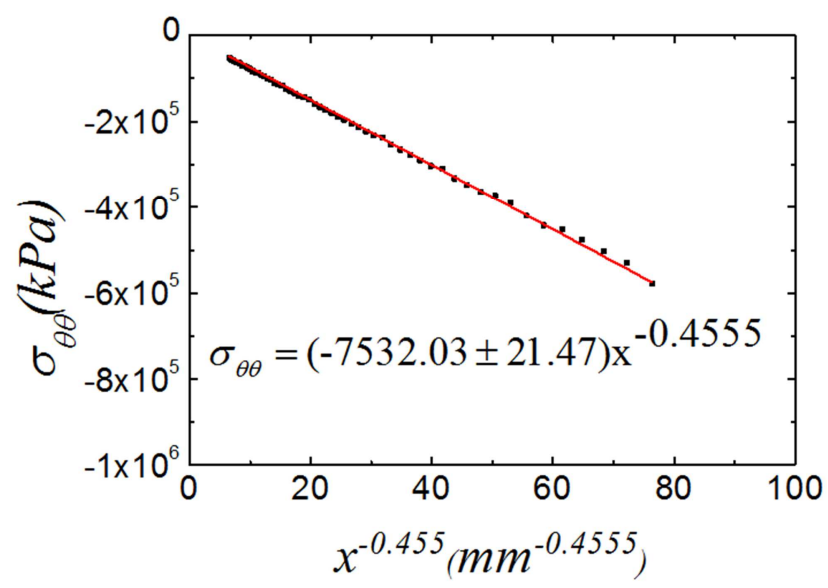

Figure 4. The first stress intensity factor $K_{\text {II }}$ can be determined from curve fitting of the stress and the distance to the corner.

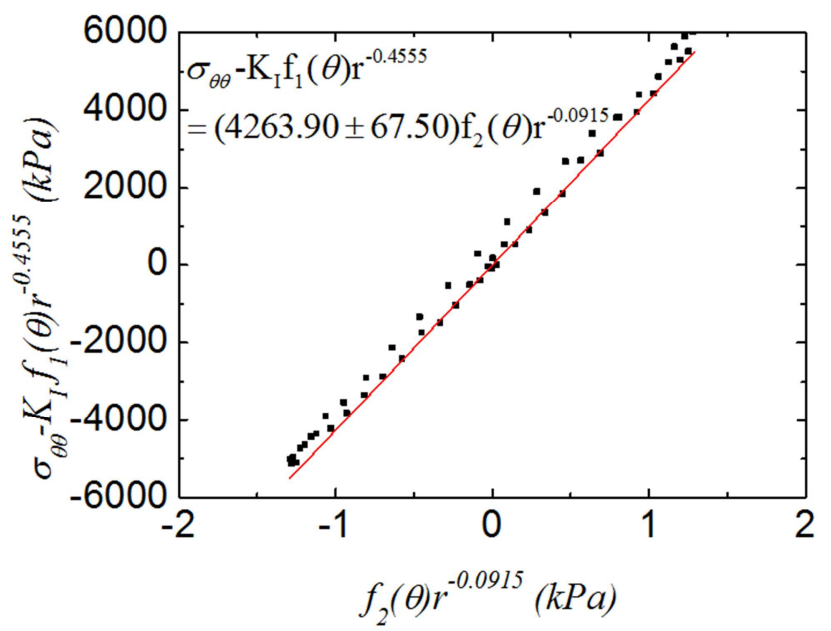

Figure 5. The second stress intensity factor $K_{\text {II }}$ can also be determined by curve fitting. 


\subsection{Generalized Stress Around a Sharp Corner}

In order to illustrate the generality of the normalization approach using $x_{0}$ and $\tau_{0}$, the stress fields under 3 different loading conditions and system dimensions, as shown in Fig. 3 , were computed. By repeating the calculation procedures in section 3.2 , the length term scale $x_{0}$ and stress term $\tau_{0}$ can be obtained. Figure 6 shows that with elastic analysis the three cases shown in Fig. 3 give the same normalized normal and shear stresses along the interface. The contact shear stress and contact pressure are also modeled when there is a normalized yield stress of $\sigma_{y} / \tau_{0}=25$, showing that all the plastic and elastic curves collapse outside the plastic zone. So the dimensions and loading conditions do not influence the normalized result. In other words, the normalized slip results will be representative of various combinations of loading conditions and dimensions as long as they have the same $K$ field.

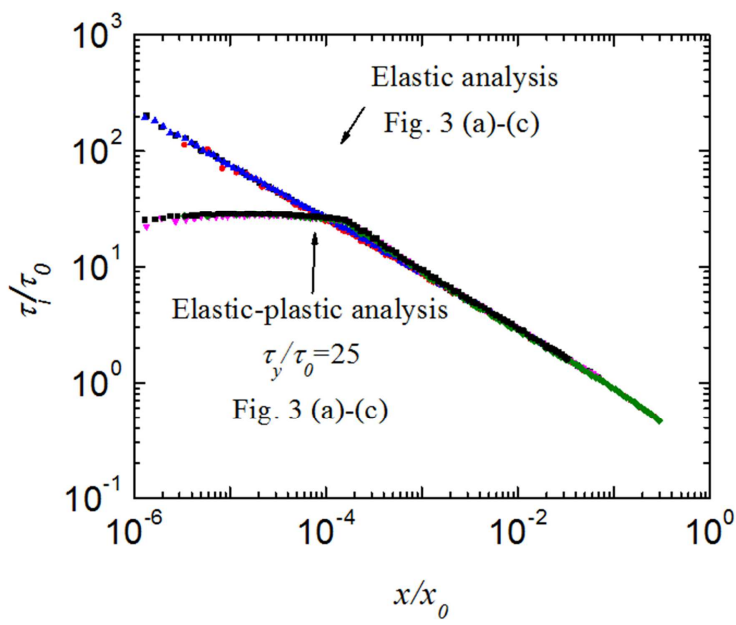

(a)

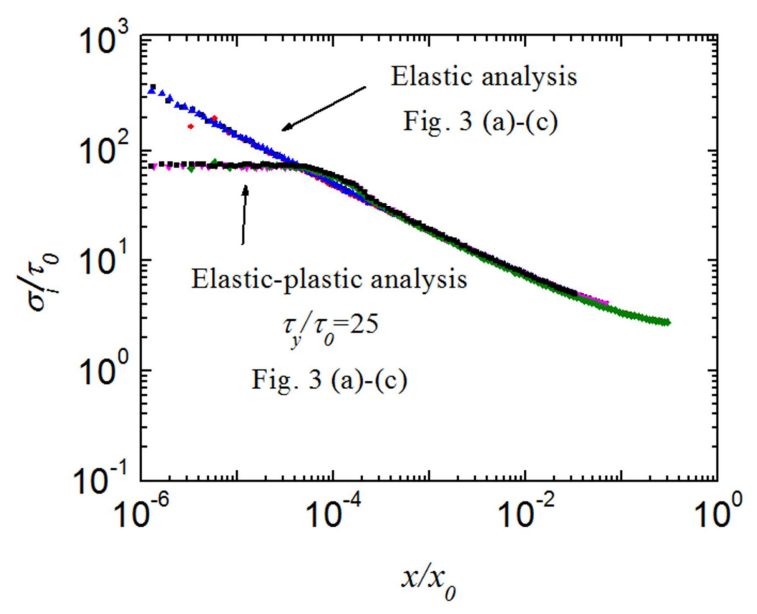

(b)

Figure 6. The curves of (a) normalized contact normal stress and (b) normalized contact shear stress as a function of the normalized coordinate $x / x_{0}$. These curves collapse into a single curve, suggesting that with normalized values the analysis would be representative of different loading conditions and system dimensions.

\section{Conclusions}

The slip behaviors around a sharp corner is highly sensitive to the specific loading condition, corner angle, dimensions and local yielding. In many applications, only small portion of the contact interface has slip or yielding. In this case, the contact behaviors generally follows the elastic solution. In this paper, a generalized form of the elastic stress filed is formulated. It is found this generalized form can well describe the stress field even if a small slip or plastic yielding zone is present around the corner. This generalized solution is independent on the loading condition or dimensions and therefore can be easily scaled to represent various boundary conditions or geometries.

\section{References}

[1] Hoeppner, D. W., Fretting fatigue case studies of engineering components. Tribology International, 2006. 39(10): p. 1271-1276.

[2] He, J. and F.-G. Yuan, Damage identification for composite structures using a cross-correlation reverse-time migration technique. Structural Health Monitoring, 2015.

[3] He, J. and F.-G. Yuan. An enhanced CCRTM (E-CCRTM) damage imaging technique using a $2 \mathrm{D}$ areal scan for composite plates. 2016.

[4] Cai, W., J. Chan, and D. Garmire, 3-Axes MEMS Hall-Effect Sensor, in 2011 IEEE Sensors Applications Symposium. 2011. p. 141-144.

[5] Cai, W. and L. Gouveia, Modeling and simulation of Maximum power point tracker in Ptolemy. Journal of Clean Energy Technologies, 2013. 1(1): p. 4.

[6] Cai, W., X. Cui, and X. Zhou, Optimization of a GPU Implementation of Multi-dimensional RF Pulse Design Algorithm, in International Conference on Bioinformatics and Biomedical Engineering 2011. 2011.

[7] Cai, W., L. Huang, and N. Wu, Class E Power Amplifier for Wireless Medical Sensor Network. International Journal of Enhanced Research in Science, Technology \& Engineering, 2016. 5(4): p. 6.

[8] Wang, J., et al., An Innovative Two-Stage Reheating Process for Wrought Aluminum Alloy During Thixoforming. Metallurgical and Materials Transactions A, 2015. 46(9): p. 4191-4201.

[9] Wang, J. J., et al., Alloy development and reheating process exploration of $\mathrm{Al}-\mathrm{Si}$ casting alloys with globular grains for thixoforming. Journal of Materials Research, 2016. 31(16): p. 2482-2492.

[10] Zhang, J., et al., An optimized cross-linked network model to simulate the linear elastic material response of a smart polymer. Journal of Intelligent Material Systems and Structures, 2015.

[11] Zhang, J., et al., A novel statistical spring-bead based network model for self-sensing smart polymer materials. Smart Materials and Structures, 2015. 24(8): p. 085022.

[12] $\mathrm{Hu}, \mathrm{Z}$., et al., Effect of plastic deformation on the evolution of wear and local stress fields in fretting. International Journal of Solids and Structures, 2016. 82: p. 1-8. 
[13] Hu, Z., M. D. Thouless, and W. Lu, Effects of gap size and excitation frequency on the vibrational behavior and wear rate of fuel rods. Nuclear Engineering and Design, 2016. 308: p. 261-268.

[14] Hu, Z. and J. W. Pratt. The Environmental and Economic Impact of IGCC in China, With Comparison to Alternative Options. in Proceeding of ASME International Conference on Energy Sustainability, Volume 1. 2010. Phoenix, Arizona.

[15] Banerjee, N. and D. A. Hills, Analysis of stick-slip and contact-edge behaviour in a simplified fretting fatigue test. The Journal of Strain Analysis for Engineering Design, 2006. 41: p. 183-192.

[16] Tan, L., A. Acharya, and K. Dayal, Modeling of slow timescale behavior of fast molecular dynamic systems. Journal of the Mechanics and Physics of Solids, 2014. 64: p. 24-43.

[17] Tan, L. and K. Bhattacharya, Length scales and pinning of interfaces. Philosophical Transactions of the Royal Society A: Mathematical, Physical and Engineering Sciences, 2016. 374(2066).

[18] Hu, Z., W. Lu, and M. D. Thouless, Slip and wear at a corner with Coulomb friction and an interfacial strength. Wear, 2015. 338-339: p. 242-251.

[19] $\mathrm{Hu}, \mathrm{Z}$., et al., Simulation of wear evolution using fictitious eigenstrains. Tribology International, 2015. 82, Part A(0): p. 191-194.

[20] He, J. and F.-G. Yuan, Lamb-wave-based two-dimensional areal scan damage imaging using reverse-time migration with a normalized zero-lag cross-correlation imaging condition. Structural Health Monitoring, 2016.

[21] Lu, W., et al., CASL Structural Mechanics Modeling of Gridto-Rod Fretting (GTRF). JOM, 2016. 68(11): p. 2922-2929.

[22] Vingsbo, O. and S. Söderberg, On fretting maps. Wear, 1988. 126(2): p. 131-147.

[23] He, J. and F.-G. Yuan, Lamb wave-based subwavelength damage imaging using the DORT-MUSIC technique in metallic plates. Structural Health Monitoring, 2016.

[24] Tan, L., A. Acharya, and K. Dayal, Coarse variables of autonomous ODE systems and their evolution. Computer Methods in Applied Mechanics and Engineering, 2013. 253: p. 199-218.

[25] Zhou, Z. R., et al., Progress in fretting maps. Tribology International, 2006. 39(10): p. 1068-1073.

[26] Gu, H., Molecular Dynamics Study on Mechanical Properties and Interfacial Morphology of an Aluminum Matrix Nanocomposite Reinforced by $\beta$-Silicon Carbide Nanoparticles. Journal of Computational and Theoretical Nanoscience, 2009. 6: p. 12.

[27] Gu, H., A Technical Brief - Applications of Conductive Polymer Composites in Oil and Gas Industry, in Petroleum and Petrochemical Technical Symposium 2015: Huston, TX.

[28] Peng, Y., et al., A comprehensive computational model of sound transmission through the porcine lung. The Journal of the Acoustical Society of America, 2013. 134(5): p. 41214121.

[29] Peng, Y., et al. Experimental Study on Gastric Electrophysiology Signal by Electroacupuncture at Zusanli Point on Rabbits. in 2008 2nd International Conference on
Bioinformatics and Biomedical Engineering. 2008.

[30] Peng, Y., et al., Sound transmission in the chest under surface excitation: an experimental and computational study with diagnostic applications. Medical \& Biological Engineering \& Computing, 2014. 52(8): p. 695-706.

[31] Peng, Y., et al., Sound transmission in porcine thorax through airway insonification. Medical \& Biological Engineering \& Computing, 2016. 54(4): p. 675-689.

[32] Peng, Y., et al. Chest Response to Vibratory Excitation: Advances Beyond Percussion in ASME 2012 International Mechanical Engineering Congress and Exposition. 2012. Houston, TX.

[33] Wang, J., A. B. Phillion, and G. Lu, Development of a viscoplastic constitutive modeling for thixoforming of AA6061 in semi-solid state. Journal of Alloys and Compounds, 2014. 609: p. 290-295.

[34] Narayanunni, V., H. Gu, and C. Yu, Monte Carlo simulation for investigating influence of junction and nanofiber properties on electrical conductivity of segregated-network nanocomposites. Acta Materialia, 2011. 59(11): p. 4548-4555.

[35] Wang, J., et al., Viscosity estimation of semi-solid alloys based on thermal simulation compression tests. International Journal of Materials Research, 2013. 104(3): p. 255-259.

[36] Ma, X. and H.-J. Shi, On the fatigue small crack behaviors of directionally solidified superalloy DZ4 by in situ SEM observations. International Journal of Fatigue, 2012. 35(1): p. 91-98.

[37] Ma, X. and H.-J. Shi, In situ SEM studies of the low cycle fatigue behavior of DZ4 superalloy at elevated temperature: Effect of partial recrystallization. International Journal of Fatigue, 2014. 61: p. 255-263.

[38] Ma, X., et al., Influence of surface recrystallization on the low cycle fatigue behaviour of a single crystal superalloy. Fatigue \& Fracture of Engineering Materials \& Structures, 2015. 38(3): p. 340-351.

[39] Gu Jiansheng, et al., Effect of Structural Relaxation on Hardness and Shear Band Features of $\mathrm{Zr}$ (64.13) Cu (15.75)Ni (10.12)Al (10) Bulk Metallic Glass During Indentation. Rare Metal Materials and Engineering, 2008. S4.

[40] Zhang, J., et al., Bonding of alumina and metal using bulk metallic glass forming alloy. International Journal of Modern Physics B, 2009. 23(06n07): p. 1306-1312.

[41] Churchman, C. M. and D. A. Hills, General results for complete contacts subject to oscillatory shear. Journal of the Mechanics and Physics of Solids, 2006. 54(6): p. 1186-1205.

[42] Parmigiani, J. P. and M. D. Thouless, The effects of cohesive strength and toughness on mixed-mode delamination of beamlike geometries. Engineering Fracture Mechanics, 2007. 74(17): p. 2675-2699.

[43] Sills, R. B. and M. D. Thouless, The effect of cohesive-law parameters on mixed-mode fracture. Engineering Fracture Mechanics, (0).

[44] Giannakopoulos, A. E., T. C. Lindley, and S. Suresh, Aspects of equivalence between contact mechanics and fracture mechanics: theoretical connections and a life-prediction methodology for fretting-fatigue. Acta Materialia, 1998. 46(9): p. $2955-2968$. 
[45] Barber, J. R., Elasticity. 3rd ed. 2010, Dordrecht, NY: Springer.

[46] Bogy, D. B., Two Edge-Bonded Elastic Wedges of Different Materials and Wedge Angles Under Surface Tractions. Journal of Applied Mechanics, 1971. 38(2): p. 377-386.

[47] Kogut, L. and I. Etsion, A Semi-Analytical Solution for the Sliding Inception of a Spherical Contact. Journal of Tribology, 2003. 125(3): p. 499-506.

[48] Zhang, J., et al., Crack initiation and fatigue life prediction on aluminum lug joints using statistical volume element-based multiscale modeling. Journal of Intelligent Material Systems and Structures, 2013. 24(17): p. 2097-2109.

[49] Zhang, J., J. Johnston, and A. Chattopadhyay, Physics-based multiscale damage criterion for fatigue crack prediction in aluminium alloy. Fatigue \& Fracture of Engineering Materials \& Structures, 2014. 37(2): p. 13.
[50] Bay, N. and T. Wanheim, Real area of contact and friction stress at high pressure sliding contact. Wear, 1976. 38(2): p. 201-209.

[51] Flicek, R., D. A. Hills, and D. Dini, Progress in the application of notch asymptotics to the understanding of complete contacts subject to fretting fatigue. Fatigue \& Fracture of Engineering Materials \& Structures, 2013. 36(1): p. 56-64.

[52] Hills, D. A. and D. Dini, Characteristics of the process zone at sharp notch roots. International Journal of Solids and Structures, 2011. 48(14-15): p. 2177-2183.

[53] Williams, M. L., Stress singularities resulting from various boundary conditions in angular corners of plate in extension. ASME Journal of Applied Mechanics, 1952. 19: p. 526-528. 